\title{
stockages souterrains d'hydrocarbures: sécurité et protection de l'environnement
}

\author{
par \\ A. C. Lacoste \\ Ministère de I'Industrie \\ P. Bérest \\ Ministère de I'Industrie
}

RESUME - Le stockage souterrain des hydrocarbures présente, sur le plan de la sécurité et de l'environnement, des avantages considérables. Certains problèmes subsistent néanmoins, qui concernent en particulier la protection de l'espace souterrain. L'organisation du controle et de la surveillance de ces ouvrages par I'administration est décrit ; trois exemples illustrent les solutions qui ont pu être apportées aux difficultés subsistantes.

\section{INTRODUCTION}

La consommation française de produits pétroliers a vu sa croissance se réduire depuis plusieurs années; les volumes impliqués restent néanmoins considérables (108 millions de tonnes de pétrole et 24 milliards de mètres cubes normaux de gaz naturel). Ces quantités énormes impliquent des capacités de stockage proportionnées, pour des raisons qui peuvent être variées :

- pour le pétrole et ses dérivés liquides (naphta, fuel, essence etc...) il s'agit avant tout de protéger l'ensemble de I'économie de la vulnérabilité à une interruption de tout ou partie de nos approvisionnements : à cette fin le stock minimal, adopté par l'ensemble des pays occidentaux, est de trois mois de consommation.

- pour le gaz naturel, le stockage permet un ajustement entre l'approvisionnement, constant au cours de l'année, et la consommation, fortement fluctuante en raison de la part importante qu'y occupe le chauffage ; la réalisation de cet objectif exige le stockage de deux mois de consommation moyenne environ.

- pour les produits chimiques de base (éthylène, propylène, ammoniac etc...) ou les gaz de pétrole liquéfiés (butane et propane) la fonction du stock est plus classique : ces quelques produits résultent de l'activité d'un petit nombre de grosses unités et constituent la matière première de milliers de produits en aval : la fabrication de ces derniers ne doit pas être trop rigidement dépendante d'un fonctionnement continu des unités amont.

Le stockage de produits énergétiques apparait donc comme une nécessité absolue. Un décalage entre les besoins et la capacité réalisée conduirait à un choix dont les deux termes seraient inadmissibles :
- la réponse à un approvisionnement déficitaire ou à une demande excédentaire par le rationnement, qui signifiera suivant les cas le chomage technique ou l'inconfort grave des usagers domestiques.

- le report provisoire de la consommation sur une autre source d'énergie : cette solution implique le double équipement des usagers, et ne fait que déplacer le problème de constitution de stocks : cette solution colteuse peut donner une souplesse utile, mais n'est pas à l'évidence suffisante.

Si le stockage apparait nécessaire, il est clair en même temps qu'il soulève par nature des difficultés sur le plan de l'environnement et de la sécurité. Il résulte directement en effet de la nature et de la quantité des produits stockés deux problèmes :

- un problème de volume : les chiffres précédents montrent qu'il faut stocker environ 30 millions de mètres cubes de pétrole et 4 milliards de mètres cubes normaux de gaz naturel. Imaginons un instant que la seule solution techniquement envisageable soit de stocker ces produits dans de grandes cuves métalliques analogues aux anciens gazomètres dont quelques exemplaires sont encore visibles en Région Parisienne : en donnant à ces cuves 20 metres de haut et 50 mètres de diamètre, il en faudrait encore 750 pour stocker le pétrole et ... 300000 pour stocker le gaz naturel !

- un problème de sécurité : ces produits sont susceptibles de braler ou d'exploser ; c'est précisément pour les utiliser de cette manière, dans une chaudière ou un moteur de véhicule, que ces produits sont recherchés. De plus la plupart de ces produits sont toxiques, à partir d'une certaine concentration dans l'air ou dans l'eau ; enfin l'obstacle du volume, rappelé plus haut, conduit à stocker certains d'entre eux sous pression, ce qui constitue en soi un danger supplémentaire. 
Les grands stockages de produits énergétiques -ou chimiques- constituent donc un exemple particulièrement démonstratif des oppositions qui peuvent naitre entre la nécessité de l'équipement industriel du pays et le souci de préservation de l'environnement. Nous essaierons de montrer que le stockage souterrain parait le moyen de passer un bon compromis entre ces deux exigences. Mais il est d'abord nécessaire de distinguer dans les problèmes d'environnement deux aspects.

Du point de vue de l'économie globale de l'environnement, le stockage souterrain présente un avantage considérable : les installations visibles, celles qui peuvent générer bruits, odeurs, ou être simplement inesthétiques, sont réduites au minimum. Un grand aquifère de stockage de gaz naturel occupe une trentaine d'hectares de terrains de surface, dont les deux tiers sont composés par les dégagements nécessaires autour des têtes de puits. Un tel aquifère permet, pendant la saison froide, d'alimenter le cinquième de la France. L'économie est considérable : il n'en reste pas moins que ces équipements peuvent constituer une gêne pour les habitants du voisinage immédiat ; il est normal que des dispositions soient prises pour diminuer cette gêne, et légitime que les procédures administratives permettent aux habitants de participer à la définition de ces dispositions.
Cet aspect n'est toutefois aucunement spécifique des stockages souterrains ; à tout prendre, les problèmes soulevés ont ici moins aigus que pour beaucoup d'autres installations industrielles.

Le second aspect est plus caractéristique et concerne la sécurité de l'environnement devant un risque majeur, tel qu'incendie ou explosion, ou devant une pollution de la surface ou du sous-sol par les hydro carbures. Là aussi, le stockage souterrain parait présenter des avantages remarquables : c'est cet aspect, plus original, qui fera l'objet de la suite de cet article.

\section{LES TECHNIQUES DE STOCKAGE DES HYDROCARBURES DANS LE SOUS-SOL}

Dans le cadre de cet article, il n'est pas possible de discuter dans le détail les diverses techniques utilisables. Le tableau joint, à double entrée, donne la liste des stockages existants, par nature de produits et par technique de création, en France.

STOCKAGES SOUTERRAINS FRANCAIS (1979)

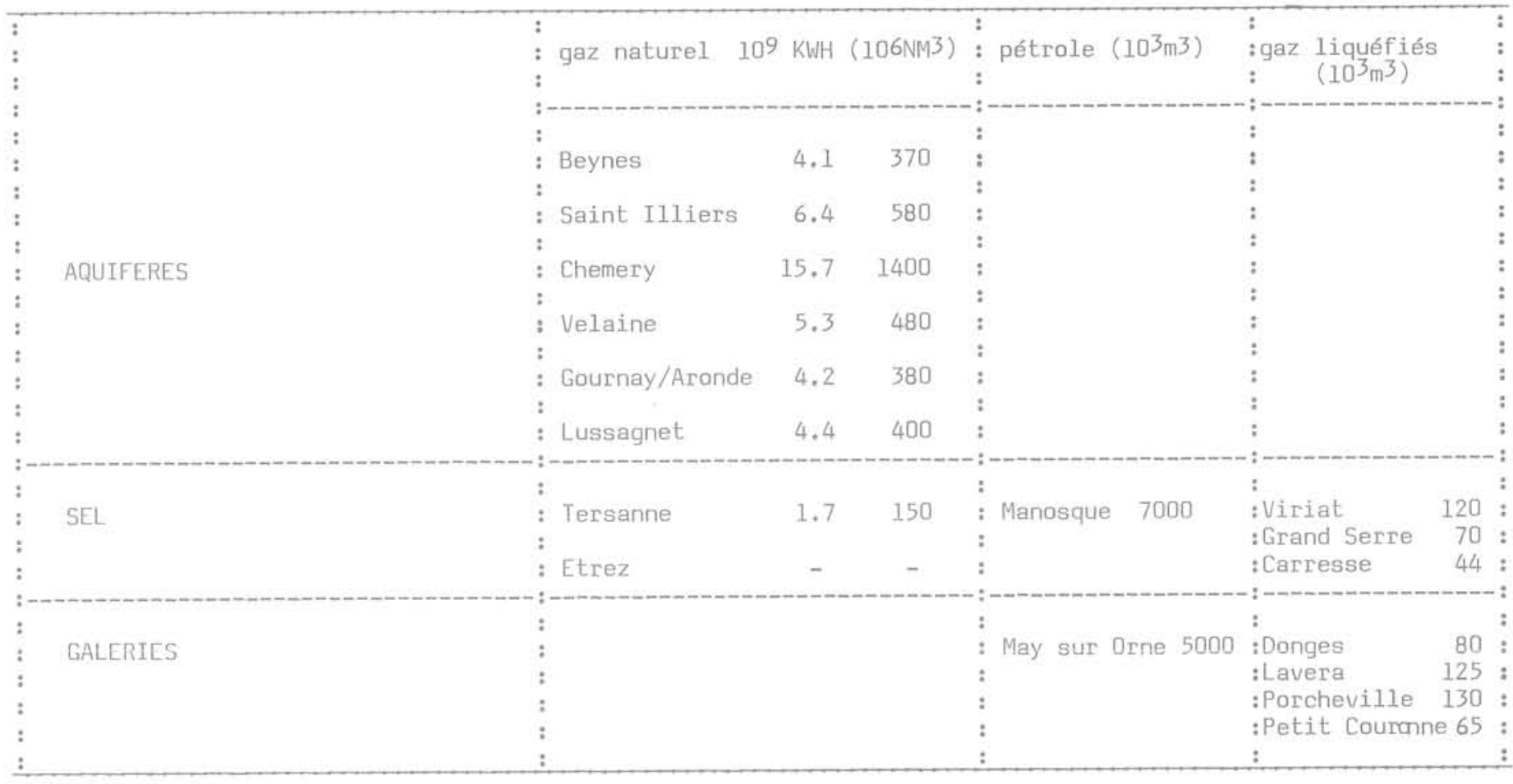


On peut rapidement rappeler les caractéristiques des trois techniques principales :

- dans le stockage en aquifère, on reproduit un gisement naturel de gaz en choisissant un site qui en rassemble toutes les conditions : un niveau suffisamment poreux et perméable, surmonté d'une couche épaisse imperméable de forme bombée, de sorte que le gaz soit piégé comme sous une cloche dans la partie haute de la structure.

- dans le stockage en cavité saline, on utilise la remarquable solubilité du sel gemme pour creuser dans les gisements profonds de sel des cavités de grandes dimensions par simple circulation d'eau douce. Cette technique est utilisable pour tous les hydrocarbures, liquides, gazeux ou liquéfiés.

- dans le stockage en galeries minées, on creuse par des moyens classiques des galeries analoques à des galeries minières ou des tunnels ; mais ces galeries sont fermées, à l'exception des tubes qui permettent la circulation des fluides stockés. Le coot de creusement est relativement élevé, ce qui rend cette technique attractive seulement pour les produits liquides ou liquéfiables.

Ces techniques ont été très largement utilisées dans le monde entier, Il existe aux U S A plus de 400 aquifères (certains d'entre eux sont constitués dans d'anciens gisements de gaz, ce qui souligne 1'analogie) et aux environs de 2000 cavités de stockage dans le sel (pour une soixantaine en France). Dans les pays du nord de l'Europe, la remarquable résistance des roches du bouclier scandinave a rendu systématique l'utilisation de galeries minées de très grandes dimensions. En R F A, l'essentiel du stockage souterrain est réalisé en aquifère ou dans le sel. Le tableau joint montre que la variété de la géologie du sous-sol français a permis une répartition assez équilibrée des différentes techniques.

\section{Avantages généraux du stockage souterrain -}

Le stockage souterrain doit être comparé, du point de vue de la sécurité et de la protection de l'environnement, à la solution alternative qui est le stockage en cuves métalliques à la surface du sol. Le stockage souterrain présente par nature de nombreux avantages.

- l'emprise au sol : un stockage en aquifère comporte en surface une quarantaine de têtesde puits, de dimension très faible, mais qui sont entourées d'une zone dégagée d'un demi hectare ; en y ajoutant une station d'une dizaine d'hectares, I'emprise totale est de 1'ordre de la trentaine d'hectares ; un stockage en gazomètres d'un demi milliard de mètres cubes en accuperait plusieurs dizaines de milliers. Les proportions sont moindres pour un stockage de liquide mais restent importantes.

- vis à vis des agressions extérieures : un stockage aérien de capacité équivalente comporte un grand nombre de cuves et offre donc aux agressions extérieures (chute d'avion, incendie initié par une autre installation industrielle etc...) une prise considérable. Le stock souterrain est au contraire protégé par une "paroi" naturelle qui peut atteindre plusieurs centaines de mètres.
- du point de vue des conséquences d'un accident : une déchirure d'une cuve métallique met l'ensemble du stockage en contact avec l'oxygène de l'air, qui est le comburant naturel des produits stockés. Au contraire la masse des produits stockés sousle sol est séparée de l'atmosphère par un isolant de plusieurs centaines de mètres. Si on imagine donc un accident symétrique à la déchirure d'une cuve, soit la rupture d'une tête de puits (on verra plus loin comment on peut l'éviter), on s'aperçoit que l'incendie éventuel est localisé en un point précis, ce qui dans la plupart des cas réduira sensiblement les conséquences et permettra une intervention adéquate,

- un avantage plus particulier est lié au stockage sous pression. Certains produits, comme les GPL, présentent un grand volume à l'état gazeux ; il est donc intéressant de les stocker à l'état liquide, mais cela nécessite d'augmenter leur pression, comme on le fait dans les bouteilles de butane à usage domestique. La prévention des dangers présentés par les fortes pressions peut être colteuse ; au contraire, lorsque l'on descend sous la surface du sol, la pression de l'eau qui imbibe les roches augmente avec la profondeur : sous terre, les fortes pressions deviennent une caractéristique naturelle des fluides et les difficultés liées au contraste entre la pression ambiante et la pression de stockage se réduisent.

Ces avantages généraux sont considérables. Il est d'ailleurs remarquable de constater que les pays qui attachent traditionnellement une importance considérable à la sécurité civile sous tous ses aspects et en particulier dans la perspective d'un affrontement militaire, privilégient le stockage souterrain par rapport à tout autre technique : c'est la cas en particulier des pays scandinaves. De même certains pays exportateurs de pétrole du Moyen Orient se dotent d'une capacité de stockage de produits raffinés, qui n'est à l'évidence pas motivée par des problèmes d'approvisionnement.

Ce préjugé très favorable a une conséquence étonnante: à la lecture des comptes rendus des congrès internationaux consacrés au stockage souterrain, on constate que ces techniques apparaissent si sares que les risques résiduels ne font pas I'djet d'un examen systématique ou même, dans certains pays, d'un contrôle particulier par l'administration; au contraire des stockages aériens, à la sécurité desquels la plus grande attention est en général portée.

La situation française s'explique sans doute par des traditions différentes et a conduit dès l'origine les sociétés à porter un intérêt particulier à ce problème ; c'est peut-être un des éléments qui explique la réputation de la technique française dans ce domaine, et les succès qu'elle a pu rencontrer à l'étranger. Les risques résiduels méritent en effet une attention soigneuse :

- les inconvénients présentés par les stockages aériens se retrouvent posés, à une échelle très réduite, par les têtes de puits qui sont les seules traces à la surface du stockage souterrain, et donc un point vulnérable. Ces têtes doivent être largement calcukes et protégées efficacement.

- des problèmes spécifiques à l'environnement souterrain apparaissent. Il s'agit pour l'essentiel de la conservation de la stabilité mécanique des cavités, 
de la prévention des fuites souterraines et de la protection des risques d'éruption (cette dernière notion recouvre, au sens large, les conséquences d'une défaillance importante de 1 'étanchéité des canalisations verticales qui permettent la circulation des fluides entre le stockage proprement dit et la surface).

Organisation du controle et de la surveillance des stockaqes souterrains -

Le contrôle et la surveillance sont assurés pour l'essentiel par le ministère de l'industrie ; leur définition est largement inspirée des principes qui quident depuis plusieurs siècle l'exercice de la police des mines. Ces principes s'efforcent de combiner :

- d'une part un contrôle stricto sensu, qui s'applique à vérifier la conformité des ouvrages à un ensemble de dispositions; ce contrôle est assorti de pouvoirs de sanction.

- d'autre part une politique d'incitation, qui exige un suivi attentif de l'évolution de l'état de l'art et une haute qualification des agents chargés du contrôle.

En effet le stockage souterrain est une activité relativement nouvelle ; la technologie y évolue de manière constante ; il serait absurde d'enfermer ce développement dans des règles techniques de détail, contraignantes et fiqées. Ceci implique :

- une réglementation souple, mais qui échelonne les autorisations administratives aux grandes étapes de la réalisation du stockage (par exemple, forage, essais de forage, injection expérimentale, essais d'étanchéité, autorisation de mise en service), ce qui permet de ponctuer chaque phase par un examen technique attentif.

- un dialogue permanent avec les concepteurs et exploitants de stockages souterrains.

- une orqanisation convenable de l'administration : le controle des stockages est assuré par les directions interdépartementales de l'industrie ; mais une direction centrale, la direction de la qualité et de la sécurité industrielles (DQSI) est chargée de coordonner et d'animer les activités du ministère de l'industrie en matière de sécurité des stockages, en relation avec les directions chargées de définir, par produits, les objectifs de la politique de stockage (direction des hydrocarbures ; direction du gaz, de I'électricité et de charbon) ; la DQSI organise la formation spécifique des agents charqés du controle, elle définit et finance un programme d'études techniques d'intérêt général. L'importance accordée à ces problèmes a été soulignée récemment par la création, par le Ministre de l'Industrie, d'une Commission de Sécurité des Stockages Souterrains.

Quelques problèmes liés à la sécurité et à la protection de l'environnement -

I1 n'est pas possible, dans le cadre du présent article, de présenter exhaustivement les problèmes de sécurité et d'environnement posés par les stockages souterrains et les solutions qui ont pu y être apportés. Il serait pourtant dommage, dans le cadre de journées organisées par des géotechniciens, de ne pas offrir à la discussion quelques exemples de problèmes importants et relativement originaux soulevés par le caractère souterrain des ouvrages de stockage; nous avons choisi trois d'entre eux qui ont paru particulièrement caractéristiques.

La Stabilité Mécanique : l'exemple des cavités dans le sel -

La planche jointe présente unecape verticale de diverses cavités lessivées dans le sel gemme, en France et à l'étranger.

EXEMPLES DE CAVITES SALINES DANS LE MONDE

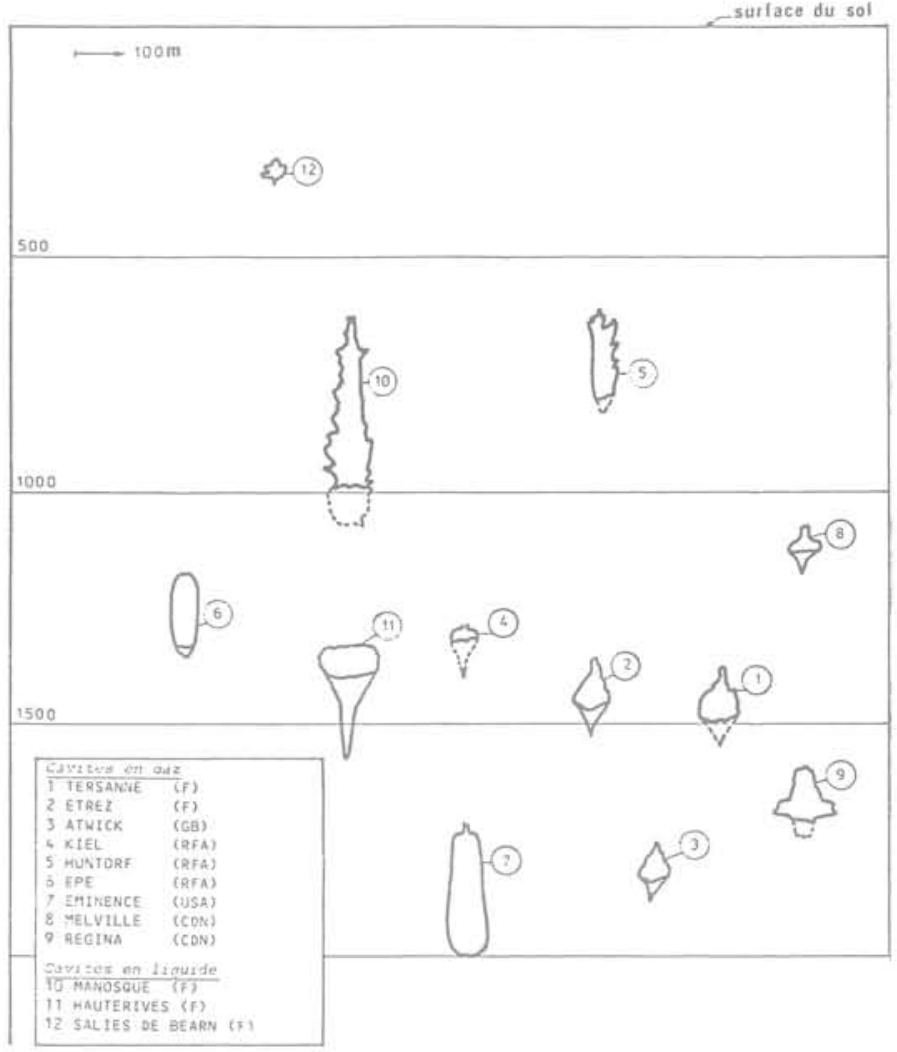


Ces cavités ont des dimensions considérables; elles paraissent néanmoins petites à l'échelle de leur recouvrement, de sorte que le risque d'un effondrement brutal se propageant jusqu'à la surface n'est pas à prendre en compte : au moins pour les dimensions et les espacements respectés jusqu'à ce jour, 1'expérience acquise à travers deux milliers de cavités de ce type réalisées dans le monde élimine la perspective d'un tel accident.

Des désordres localisés au voisinage des cavités $n^{\prime}$ en pourraient pas moins avoir des conséquences graves, spécialement si s'établissaient des communications entre la cavité et des formations non selifères ; Ies produits s'échapperaient alors de la cavité ou, peut etre pire, de l'eau douce pénéterait massivement dans le gisement salifère.

Le problème posé est tout à fait original ; cette originalité tient à une propriété très remarquable : le sel gemme est incapable de supporter durablement un écart important à l'état de contraintes isotrope. Ce fait, qui explique le caractère particulier de la tectonique salifère, est souvent mal pris en compte; il conduit à des erreurs d'interprétation et explique le tour polémique que prennent souvent les discussions des spécialistes, Du point de vue de l'administration, deux problèmes se posaient :

- la pression intérieure, la profondeur, la géométrie de la cavité sont a priori les paramètres essentiels de la stabilité. Peut-on établir pour ceux-ci les règles assurant la stabilité d'une cavité ?

- plusieurs projets prévoyaient 1'implantation de dizaines de cavités dans le même gisement. Quel espacement minimal devait on imposer entre deux cavités voisines?

Comme souvent les réponses à ces questions ont évolué dans le temps, en particulier lorsque les prédictions des calculs ont été soumises à l'épreuve de l'expérience.

Dans un premier temps, une expérimentation systématique en laboratoire a été effectuée sur des échantillons prélevés par carottage, pour déterminer les paramètres du comportement du sel dans h perspective de ]'utilisation du modèle élastoplastique parfait. Les progrès faits à l'époque dans le domaine du calcul, en particulier par l'utilisation des résolutions par éléments finis, permettaient d'introduire ces paramètres dans des modèles rendant compte du comportement de l'ensemble de la structure.

Les résultats se résumaient ainsi :

- aux pressions envisagées, les cavités perdraient un volume de l'ordre de quelques pour cent.

- la jonction des zones plastiques de deux cavités voisines devait être évitée : ceci conduisait à des règles de dimensionnement, variables suivant les sites, mais que l'on peut grossièrement résumer de la manière suivant : la distance entre axes de cavités voisines doit être de trois à quatre fois le diamètre maximum des cavités.

En 1980, après une dizaine d'années d'existence de cavités dans le sel, l'expérience s'est enrichie de nombreuses constatations:
- les cavités sont restées globalement stables; aucun mouvement même minime de la surface du sol n'a été enregistré. Toutefois, les cavités profondes remplies de gaz de Tersanne (Drôme) ont vu leur volume diminuer plus fortement que prévu. La mesure du volume des cavités en gaz est particulièrement délicate, ce qui a conduit I'exploitant, Gaz de France, à mettre au point des techniques originales de traçages à I'hydrogène puis, à la demande de l'administration, à remplir une cavité de saumure pour y effectuer une mesure par sonar. Une perte de volume de $25 \%$ environ a été ainsi constatée.

- les mesures de caractéristiques mécaniques en laboratoireont été profondément remises en cause. Ces mesures posent, dans le cas du sel gemme, des problèmes de mode opératoire particulier ; de plus, les comportements différés du sel gemme ont une importance prépondérante : des essais de fluage de longue durée (plusieurs mois) ont montré que les caractéristiques réelles du sel gemme avaient été surestimées.

- les mesures in situ sur des cavités ont été développées par l'administration et par les sociétés concernées. Elles ont mis également en évidence le cornportement visqueux du sel gemme.

Toutes les conclusions n'ont pas été tirées de ce très complet ensemble d'études ; néanmoins un corps de doctrine provisoire se dégage clairement de l'expérience :

- les pertes importantes de volume par fluage ne se constatent que lorsque la différence entre le poids des terrains et la pression intérieure excède 20 à $22 \mathrm{MPa}$.

- une distance entre cavités égale à 3 ou 4 fois le diamètre maximum des cavités (suivant les conditions localesà paraît satisfaisante.

- en évitant les toits plats de grande portée, on assure une très bonne tenue de la partie supérieure de la cavité.

Le premier de ces critères a une conséquence pratique immédiate : les stockages de gaz naturel dans le sel gemme ne doivent pas être implantés trop profondément; l'optimum doit être placé quelque part entre 800 mètres et 1200 mètres. L'intérêt de cette formulation est qu'une vérification prochaine de son bien fondé est possible, plusieurs cavités ayant été récemment implantées, en Grande Bretagne et en R F A, à des profondeurs qui excèdent sensiblement la valeur maximale ici proposée.

\section{SECURITE DES TETES DE PUITS}

La tête de puits, où aboutissent les canalisations qui permettent la circulation des fluides entre la surface et le réservoir souterrain, est la seule partie aérienne du stockage ; c'est la seule qui peut être soumise aux agressions extérieures ; cette circonstance constitue un des avantages principaux des stockages souterrains sur le plan de la sécurité. 
La possibilité d'un accident grave lié à des circonstances extérieures (chute d'avion...) en est considérablement réduite, La probabilité de défaillance de l'équipement est faible : le matériel utilisé est d'usage courant dans l'industrie pétrolière ; sa fiabilité est donc éprouvée ; il est bien entendu très largement dimensionné pour l'usage particulier qui en est fait dans un stockage et comporte de nombreuses sécurités internes.

L'éventualité d'un accident majeur -rupture complète de la tête de puits- a néanmoins été examinée avec attention. Ses conséquences varient avec la nature des produits stockés :

(a) prenons 1'exemple d'un stockage de produit liquide (pétrole) dans une cavité dans le sel située à 1000 mètres de profondeur. Une explication préalable est nécessaire sur le mode de fonctionnement d'un tel stockage. Le sondage qui met en communication la surface du sol et la cavité est divisée en deux espaces distincts : l'un est rempli de pétrole et l'autre de saumure saturée. L'injection ou le soutirage de pétrole peut être ainsi compensé par un mouvement inverse de saumure saturée, de sorte que Ia cavité et l'ensemble du sondage sont toujours remplis de liquide.

Dans la cavité la saumure, plus lourde, occupe le fond, et le pétrole la surmonte, Observons comment la pression varie dans les deux parties du sondage quand on remonte vers la surface.

La pression des deux fluides est égale à l'interface; puis elle décroit lorsque l'on monte; la saumure étant plus lourde que le pétrole (les densités sont respectivement 1.2 et 0.9 ) la pression décroit toutefois plus vite dans l'espace rempli de saumure : au niveau de la tête de puits, la pression de la saumure devient égale à la pression atmosphérique, tandis que la pression du pétrole est, dans notre exemple, d'une trentaine de bars.

Si la téte de puits rompt, du pétrole jaillirait donc à la surface alors que la saumure descendrait dans le sondage. Le volume expulsé est plus important que celui qui résulte du simple rééquilibrage de ce gigantesque "tube en U" : la détente des fluides consécutive à une rupture occasionne une augmentation de leur volume qui se résorbe par expulsion brutale. Pour une cavité contenant 100000 mètres cubes de pétrole, la quantité expulsée est de quelques centaines de mètres cubes. Cette quantité appelle deux commentaires :

- elle est très faible en valeur relative : plus de $99 \%$ du contenu du stockage reste à l'abri d'un incendie ou d'une explosion. Pour cette raison, un calcul suffisamment large des caractéristiques de la tête de puits et de ses divers organes constitue en temps ordinaire une sécurité adaptée.

. elle reste significative en valeur absolue. On peut heureusement supprimer tout risque par une parade simple lorsque les circonstances appellent une sécurité exceptionnelle (lorsque des ouvriers travaillent sur la tête de puits par exemple) : il. suffit de vider le stockage de quelques millièmes de son contenu, en laissant descendre le sommet de la colonne de saumure, pour réduire à zéro la surpression du pétrole en tête de puits. On connait malheureusement un accident mortel, survenu à l'étranger, que cette précaution aurait semble-t-il permis d'éviter.

(b) le problème est plus aigu pour les stockages de gaz naturel (en aquifère ou en gisement de sel). Cette fois en effet, le produit étant gazeux, c'est la totalité des produits stockés qui sortirait avec un débit considérable en cas de rupture de la tête de puits. Cet accident majeur aurait heureusement de fortes chances d'etre surtout spectaculaire : si, comme 11 est probable, le jet s'enflamme spontanément, le gaz brolera en donnant des produits de combustion innofensifs ; cette gigantesque torchère s'éteindra après quelques jours, et le plus grand dommage sera le gaspillage d'une quantité importante d'énergie, qu'il sera peut être possible de réduire par une intervention appropriée.

Il n'en reste pas moins qu'un tel accident ferait courir des risques graves au personnel éventuellement présent au voisinage de la tête de puits ; enfin la situation serait plus délicate si l'allumage de la torche ne s'opérait pas spontanément. Gaz de France a donc choisi dès l'origine, en concertation avec 1 'administration, de prévoir une sécurité exọtionnelle contre ce type d'accident : tous les sondages en communication avec le stock de gaz sont équipés d'une vanne souterraine de sécurité, placée à une trentaine de mètres sous terre. Cette vanne est à sécurité positive : elle est actionnée automatiquement par une chute de pression en tête ; son principe est le même que celles des vannes utilisées dans les exploitations pétrolières offshore où, pour d'autres raisons, des précautions particulières contre les risques d'éruption doivent être prises.

De telles vannes équipent plusieurs centaines de sondages de stockages souterrains en France ; il faut souligner que, très heureusement, depuis le début de leur installation il y a une vingtaine d'années, aucune de ces vannes n'a eu l'occasion de remplir son rôle de sécurité ultime. La France est, à notre connaissance, le seul pays où les sondages en gaz naturel soient systématiquement équipés de cette manière ; d'autres pays européens envisagent d'imposer des dispositifs du même type.

(c) les stockages de produits liquéfiés présentent un cas intermédiaire, Dans le scénario le plus pessimiste, après une expulsion initiale brutale d'une fraction du volume stocké sous forme liquide, la cavité se viderait lentement, la vitesse de vaporisation étant réglée par l'apport de chaleur qu'elle nécessite. La solution de la vanne de sécurité n'est malheureusement pas directement transposable, car elle soulève ici des problèmes technologiques particuliers -au moins dans le cas des cavités lessivées en gisement de sel ra protection de la tête de puits par un ouvrage ad hoc parait, en l'état actuel de la techno].sgie, le moyen le plus efficace de compléter la sécurité ; il n'en reste pas moins que c'est sans doute pour ce cas particulier que les efforts les r.' is importants devront dans I'avenir être consentis. 
La discussion détaillée des phénomènes sort du cadre de cette note. Elle a fait 1'objet d'un examen attentif, mené conjointement par les sociétés concernées et l'administration ; cet examen a permis de dégager des règles précises, qui tiennent compte en particulier de la géométrie propre de chaque stockage. On peut retenir de ces règles des ordres de grandeur applicables en première approximation : pour une galerie en propane d'une hauteur modérée, une dépression d'une trentaine de mètres d'eau est à même d'assurer une très bonne sécurité du stockage vis à vis du maintien du confinement.

Cette valeur peut être localement modifiée, pour tenir compte de circonstances particulières ; elle constitue néanmoins un ordre de grandeur convenable. Il est regrettable que l'on ne dispose pas, dans la littérature d'origine étrangère, de données précises sur ce point. Certains textes parlent de cing mètres, ce qui nous parait beaucoup trop faible ; par contre de très bons article, d'origine suédoise ou japonaise, traitent en profondeur le problème des principes du calcul. Le deuxième point de la discussion précédent, l'existence de "l'appel d'eau" sur l'ensemble du pourtour, n'a toutefois été complétement développé qu'en France à l'initiative de l'administration; il a conduit la société Géostock à developper une méthodologie pratique appelée "détermination du coefficient de forme".

\section{CONCLUSION}

Les stockages souterrains d'hydrocarbures oonstituent la solution la plus rationnelle, dès que les volumes impliqués sont importants : ils sont moins coateux, plus discrets et plus sors que les autres formes de stockage. Dans certains cas, comme celui du gaz naturel, ces avantages suppriment pratiquement la possibilité d'alternatives.

La poursuite des options énerqétiques prises dans le domaine des hydrocarbures devrait conduire à un développement soutenu de ce type de stockage,en particulier dans les domaines du gaz naturel et des gaz de pétrole liquéfiés.

Une difficulté particulière tient à ce que les avantages incontestables de cette technique sont parfois mal perçus par l'opinion publique. Ceci tient à deux raisons au moins. La première est que le plus souvent ces stockages ne sont pas implantés dans un environnement industriel, et que les habitants des sites concernés craignent donc les nuisances d'installations pour eux inhabituelles ; ce qui parait au voisinage d'un grand port un progrès considérable contient encore une menace de nuisances dans un environnement où l'industrie est inconnue. La deuxième raison tient au caractère complexe, sinon parfois mystérieux des problèmes liés au sous-sol, qui conduit à de fréquents contresens : un quotidien national intitulait il y a quelques mois un article consacré à un projet de stockage en aquifère... "Grandes manoeuvres autour des silos à gaz".

Ces erreurs contiennent une leçon claire, sans doute valable pour bien d'autres réalisations : une information précise et complète doit être apportée. Cette tâche est de longue haleine, compte tenu de la variété des publics, de leurs connaissances, de la nature de leurs inquiétudes. L'organisation de journées géotechniques consacrées à l'environnement contribue à cette information. Il faut remercier les organisateurs d'avoir choisi comme thème d'une de ces journées 'environnement et stockage souterrain" : les géotechniciens ont beaucoup à apporter à ce débat, pour lequel nous souhaitons avoir précisé le rôle qu'y tient l'administration. 